\title{
EXCESSO DE PESO DE ADULTOS RESIDENTES EM UM AGLOMERADO URBANO SUBNORMAL
}

\author{
Excess weight in adult dwellers of a subnormal urban \\ agglomeration
}

Exceso de peso en adultos residentes de un barrio de chabolas

Artigo Original

\section{RESUMO}

Objetivo: Avaliar a prevalência de excesso de peso e possíveis fatores de risco em adultos residentes em um aglomerado urbano subnormal. Métodos: Estudo observacional do tipo transversal, baseado em uma amostra de 582 adultos, na faixa etária de 20 a 59 anos, residentes no aglomerado urbano subnormal dos Coelhos (Recife). Definiu-se excesso de peso pelo índice de massa corporal (IMC) maior ou igual a $25 \mathrm{~kg} / \mathrm{m}^{2}$. Foram avaliadas possíveis associações com idade, sexo, raça/cor e fatores socioeconômicos (escolaridade e condição de trabalho). O efeito sobre a ocorrência de excesso de peso foi estimado pelo cálculo do Odds Ratio (OR), mediante o ajuste de modelos de regressão logística simples. A precisão e significância estatística desses ORs foram avaliadas através de intervalos de 95\% de confiança e do teste de Wald. Adotou-se nível de significância de 5\%. Resultados: A prevalência de excesso de peso foi de $62,5 \%(n=364)$, sendo maior nas mulheres $(66,1 \%$; $\mathrm{n}=251)$ do que nos homens $(56,0 \% ; \mathrm{n}=113)$, com um aumento progressivo até a faixa etária de 40 a 49 anos, passando a declinar a partir de então. Nessa faixa, houve um risco de excesso de peso de 2,6 vezes. Além da idade, pertencer ao sexo feminino e não trabalhar também representaram condições de risco. Conclusão: A elevada prevalência do excesso de peso na população adulta residente no aglomerado urbano subnormal dos Coelhos se mostrou associada ao sexo, faixa etária e condição de trabalho, constituindo-se, assim, como possíveis fatores de risco do problema.

Descritores: Sobrepeso; Obesidade; Adulto; Pobreza.

\section{ABSTRACT}

Objective: To assess the prevalence of excess weight and potential risk factors in adult dwellers of a subnormal urban agglomeration. Methods: Observational cross-sectional study based on a sample of 582 adults aged 20-59 years living in the subnormal urban agglomeration of Coelhos (Recife). Excess weight was estimated by body mass index (BMI) greater than or equal $25 \mathrm{~kg} / \mathrm{m} 2$. Possible associations with age, sex, race/color and socioeconomic factors (education and employment status) were assessed. The effect on the occurrence of excess weight was estimated by calculating odds ratio (OR) and adjusting simple logistic regression models. Accuracy and statistical significance of ORs were assessed through 95\% confidence intervals and the Wald test. Significance level was set at 5\%. Results: The prevalence of excess weight was $62.5 \%(n=364)$, with higher proportions among women $(66.1 \% ; n=251)$ than men $(56.0 \% ; n=113)$, with a gradual increase up to the age group 40-49 years and a decline from then on. In this age group, the risk of excess weight was 2.6 times. In addition to age, being female and not working were also identified as risk factors. Conclusion: The high prevalence of excess weight identified in the adult population of the subnormal urban agglomeration of Coelhos was associated with sex, age and employment status, constituting, therefore, potential risk factors for the problem.

Recebido em: 24/03/2015

Revisado em: 25/05/2015

Descriptors: Overweight; obesity; adult; poverty. 


\section{RESUMEN}

Objetivo: Evaluar la prevalencia del exceso de peso y los posibles factores de riesgo en adultos residentes de un barrio de chabolas. Métodos: Estudio observacional del tipo transversal basado en una muestra de 582 adultos en la franja de edad entre 20 y 59 años, residentes de un barrio de chabolas de los Conejos (Recife). Se definió el exceso de peso por el índice de masa corporal (IMC) mayor o igual a $25 \mathrm{~kg} / \mathrm{m}^{2}$. Fueron evaluadas las posibles asociaciones con la edad, el sexo, la razalel color y los factores socioeconómicos (escolaridad y condición de trabajo). El efecto de la ocurrencia del exceso de peso ha sido estimado por el cálculo del Odds Ratio (OR) mediante el ajuste de modelos de regresión logística simple. La precisión y la significancia estadística de los ORs fueron evaluadas a través de los intervalos de 95\% de confianza y de la prueba de Wald. Se adoptó el nivel de significancia del 5\%. Resultados: La prevalencia del exceso de peso fue del $62,5 \%(n=364)$ y mayor para las mujeres $(66,1 \%$; $n=251)$ que los hombres $(56,0 \% ; n=113)$ con un aumento progresivo hasta la franja de edad entre 40 y 49 años cuando empieza a disminuir. Hubo un riesgo del exceso de peso de 2,6 veces en esta franja de edad. Además de la edad el hecho de ser mujer y no trabajar también representan condiciones de riesgo. Conclusión: La elevada prevalencia del exceso de peso de la población adulta residente de un barrio de chabolas de los Conejos se asoció al sexo, la franja de edad y la condición de trabajo, constituyéndose de esa manera, como posibles factores de riesgo del problema.

Descriptores: Sobrepeso; Obesidad; Adulto; Pobreza.

\section{INTRODUÇÃO}

Como componente da transição epidemiológica que tem caracterizado a história nosográfica da humanidade a partir da segunda metade do século XX, a transição nutricional no Brasil vem ocorrendo de forma surpreendentemente rápida, modificando os padrões de morbimortalidade ${ }^{(1-3)}$. Conceitualmente, o processo consiste na substituição do modelo hegemônico de processos infecciosos e doenças carenciais pela emergência epidêmica das doenças crônicas não transmissíveis (DCNTs), mudando, no intervalo de apenas três gerações, a natureza, a magnitude, o contexto e, portanto, as prioridades do setor saúde no Brasil e na maioria dos outros países menos desenvolvidos ${ }^{(4,5)}$.

Nesse aspecto, o sobrepeso e a obesidade se constituem como importantes fatores de risco para a morbimortalidade de populações adultas, associando-se a $63 \%$ do total global de mortes. Desse total, 78\% delas ocorreram em países de média e baixa renda ${ }^{(6)}$. Ademais, em 2010, estima-se que o sobrepeso e a obesidade resultaram em 3,4 milhões de mortes, perda de $3,9 \%$ na expectativa de vida e $3,8 \%$ de anos perdidos por incapacidade ${ }^{(7)}$.

$\mathrm{O}$ excesso de peso corporal representado pelo sobrepeso/obesidade caracteriza-se pelo acúmulo de gordura, excedendo os padrões aceitáveis de normalidade antropométrica em diferentes graus e integrando, como elemento mais comum, um complexo de comorbidades, as chamadas doenças crônicas não transmissíveis $(\mathrm{DCNTs})^{(8,9)}$. Nesse contexto, a obesidade se destaca por ser simultaneamente doença e fator associado, sendo considerada um dos mais importantes marcadores de risco para outras doenças desse grupo ${ }^{(10-12)}$.

A prevalência mundial de sobrepeso e obesidade vem apresentando um rápido e progressivo aumento nas últimas décadas, de modo que, atualmente, 2,1 bilhões de adultos estão nessa condição, o que representa quase $30 \%$ da população mundial, configurando, assim, uma verdadeira pandemia: de 1980 a 2013, o excesso de peso aumentou $27,5 \%$ entre os adultos em escala mundial ${ }^{(1,8)}$. Nos Estados Unidos, a obesidade afeta mais de 35\% dos adultos, representando o país com maior prevalência mundial $^{(5,13)}$. Segundo a World Health Organization (WHO) $)^{(14)}$, pelo menos 2,8 milhões de pessoas morrem a cada ano como resultado do excesso de peso. Estima-se que, em 2015, aproximadamente 2,3 bilhões de adultos estarão em condição de excesso de peso, dos quais mais de 700 milhões estarão obesos, sendo possível que essas estatísticas, em 2030, atinjam números de 2,6 e 1,1 bilhões de sobrepeso e obesidade, respectivamente ${ }^{(15)}$. Nos países em desenvolvimento, a tendência de aumento do excesso de peso corporal pode ser tratada como a reprodução epidemiológica do desenvolvimento histórico de países desenvolvidos, conforme documentam pesquisas que apresentam dados de tendências temporais e geográficas em alguns países da América Latina ${ }^{(16)}$.

No caso particular do Brasil, observa-se a tendência de aumento da prevalência de sobrepeso e obesidade como uma das características marcantes do processo de transição nutricional do país ${ }^{(2-4)}$. Nesse aspecto, a partir da década de 1990, o país passou a apresentar frequências mais elevadas nos estratos de renda mais baixa ${ }^{(12,17)}$. A mobilidade social dessas condições, sobretudo da obesidade, com tendência à redução no quartil de renda mais elevada e deslocamento crescente para as famílias classificadas nos estratos econômicos mais baixos, confere, por assim dizer, a característica epidemiológica marcante do processo de transição nutricional da população brasileira nas últimas três décadas ${ }^{(3,4,12)}$. A tendência da obesidade no contexto da pobreza no Brasil coloca o agravo como tema prioritário para o campo da saúde pública na atualidade, desde que suas consequências biológicas e sociais se tornaram mais complexas e até certo ponto imprevisíveis ${ }^{(1,3,18)}$. Segundo levantamento realizado pelo Ministério da Saúde em $2013^{(10)}, 68,3 \%$ dos brasileiros adultos se encontravam com excesso de peso.

Os fatores sociodemográficos (sexo, idade, raça, escolaridade e renda) vêm ganhando destaque como 
possíveis fatores de risco para o sobrepeso e obesidade, embora diferenças na prevalência ou na especificidade dessas associações mereçam ainda maior investigação nas diferentes regiões do Brasil ${ }^{(11)}$.

Segundo o Instituto Brasileiro de Geografia e Estatística $(\mathrm{IBGE})^{(19)}$, o termo "favela" é conceituado atualmente como aglomerado urbano subnormal, que consiste em áreas "dispostas de forma desordenada, densa e carente, em sua maioria, de serviços públicos essenciais". Reitera-se, assim, a distinção entre áreas com definições de normalidade e, consequentemente, anormalidade, reafirmando-as como zonas de exclusão. Esse conceito possui certo grau de generalização, de forma a abarcar a diversidade de assentamentos irregulares existentes no país, conhecidos como favela, invasão, grota, baixada, comunidade, vila, ressaca, mocambo, palafita, entre outros. De acordo com o censo populacional realizado em 2010 pelo $\operatorname{IBGE}^{(19)}$, cerca de $6 \%$ da população brasileira reside em assentamentos irregulares (aglomerados subnormais), representando quase o dobro da proporção encontrada em 1991 (3,1\%).

A preocupação com as repercussões do excesso de peso, que era uma peculiaridade epidemiológica das classes sociais mais abastadas, passa agora a ser representada nos grupos menos favorecidos ${ }^{(12,20,21)}$, o que impõe uma reflexão teórica sobre sua dinâmica e o delineamento de políticas públicas mais eficazes para a prevenção e o controle das enfermidades nesses segmentos. Nesse sentido que se fundamenta a realização do presente estudo, que pretende avaliar a prevalência de excesso de peso e possíveis fatores de risco em adultos residentes em um aglomerado urbano subnormal.

\section{MÉTODOS}

Trata-se de um estudo observacional, de caráter transversal, tendo como base de dados a pesquisa "Saúde, nutrição e serviços assistenciais numa população favelada do Recife: um estudo baseline". É um inquérito proposto para estabelecer a linha de base de um projeto de coorte com intervenção para os próximos dez anos, especificamente focado nos problemas de saúde de interesse dominante (ou prioritários) na época investigada. O estudo foi realizado no aglomerado urbano subnormal dos Coelhos, localizado no bairro da Boa Vista, no município do Recife, capital do estado de Pernambuco. A coleta de dados ocorreu no período de junho a dezembro de 2014 na população assistida pela Estratégia de Saúde da Família, na área avaliada.

Para o cálculo da amostra, tomou-se como referência um universo de 3.816 adultos com idade compreendida entre 20 e 59 anos, a partir da base de dados do Sistema de Informação de Atenção Básica (SIAB) - Recife. Foi utilizado o Módulo StatCalc - Epi Info versão 6.04 (Centers for Disease Control and Prevention, Atlanta, Estados Unidos), sendo considerada uma prevalência de 53,8\% de sobrepeso e obesidade dos adultos nessa faixa etária, na Região Metropolitana do Recife de Pernambuco, segundo a III Pesquisa de Saúde e Nutrição, realizada em Pernambuco no ano de $2006^{(22)}$. Assumiu-se um erro de estimativa de $4,5 \%$, incorporando ainda um adicional de $10 \%$ na amostra para a compensação de possíveis perdas e estratificações para comparações internas (teste de hipóteses), obtendose assim uma amostra mínima inicial de 518 participantes. $\mathrm{Na}$ amostra final, figurou um total de 582 adultos. $\mathrm{Na}$ realidade, o número de observações aqui avaliadas constitui um subconjunto da amostra representativa de pessoas com 20 anos ou mais, calculada para representar o universo de adultos da comunidade dos Coelhos. A amostragem foi do tipo probabilístico e os domicílios visitados foram indicados por sorteio aleatório simples, com probabilidade proporcional à população de cada quadra de domicílios. As diferenças de números amostrais em alguns resultados devem-se às perdas de observações, em função de questionários incompletos por inconsistência de dados.

Os dados foram obtidos por meio de questionários aplicados com o adulto responsável pelo domicílio, e as medidas antropométricas (peso e altura) foram realizadas em todos os adultos selecionados. Para tanto, foram capacitados oito entrevistadores e cinco antropometristas para a realização das atividades em campo, bem como duas supervisoras de campo, incluindo a própria pesquisadora. Realizou-se estudo piloto visando testar o instrumento de coleta e a logística de trabalho de campo, a fim de verificar a sua exequibilidade e os possíveis ajustes nos questionários ou na operação de coleta de dados. Foram coletadas informações sobre alguns fatores biológicos: sexo, faixa etária, raça/cor e fatores socioeconômicos (escolaridade, condição de trabalho).

As variáveis referentes ao estado nutricional (peso e altura) foram analisadas através do índice de massa corporal (IMC), segundo as recomendações da Organização Mundial da Saúde (OMS) ${ }^{(23)}$, sendo então classificado em baixo peso $\left(\leq 18,5 \mathrm{~kg} / \mathrm{m}^{2}\right)$, adequado $\left(\geq 18,5 \mathrm{e}<25 \mathrm{~kg} / \mathrm{m}^{2}\right)$, sobrepeso $\left(\geq 25 \mathrm{e}<30 \mathrm{~kg} / \mathrm{m}^{2}\right)$ e obesidade $\left(\geq 30 \mathrm{~kg} / \mathrm{m}^{2}\right)$. Considerou-se excesso de peso (sobrepeso e obesidade) quando o IMC era maior ou igual a $25 \mathrm{~kg} / \mathrm{m}^{2}$.

A tomada do peso deu-se em balança digital, da marca Seca $^{\circledR} 876$, com capacidade de até $250 \mathrm{~kg}$ e escala de 100 gramas, com os indivíduos descalços, usando indumentária mínima e sem qualquer objeto nos bolsos, nas mãos ou na cabeça. Para medição da altura, utilizou-se estadiômetro portátil, marca Alturaexata Ltda., milimetrado, com precisão de até $1 \mathrm{~mm}$ em toda a sua extensão. Os voluntários ficaram em posição ereta, descalços, com membros superiores pendentes ao longo do corpo. 
As medidas antropométricas foram realizadas por duplas de técnicos treinados, e para garantir a acurácia das mensurações, foram aferidas em duplicatas. Quando a diferença entre as avaliações excedia $0,5 \mathrm{~cm}$ para altura e $100 \mathrm{~g}$ para o peso, repetia-se a mensuração e anotavam-se as duas medições com valores mais próximos, sendo então utilizada a média dessas medidas.

Para cada um dos possíveis fatores de risco arrolados neste estudo, o efeito sobre a ocorrência de excesso de peso foi estimado pelo cálculo do Odds Ratio (OR), mediante o ajuste de modelos de regressão logística simples. A precisão e significância estatística desses ORs foram avaliadas através de intervalos de $95 \%$ de confiança e do teste de Wald. Em todos os testes foi adotado o nível de significância de 5\%. A análise estatística foi realizada com o auxílio de software SPSS, versão 13.0 (SPSS Inc., Chicago, Estados Unidos).
O presente estudo foi aprovado pelo Comitê de Ética de Pesquisa do Instituto de Medicina Integral Professor Fernando Figueira CEP - IMIP, com o número de protocolo 4017-14. Todos os investigados foram informados quanto à participação voluntária na pesquisa e assinaram o Termo de Consentimento Livre e Esclarecido.

\section{RESULTADOS}

Descreve-se na Tabela I a prevalência de estado nutricional na amostra de adultos (20-59 anos) segundo as classificações do IMC, detalhando, ainda, a estratificação de resultados em função de variáveis biológicas e socioeconômicas. Verifica-se que a prevalência cumulativa de sobrepeso/obesidade foi de $62,5 \%(\mathrm{n}=364)$, sendo $56 \%$ $(n=113)$ entre os homens e $66,1 \%(n=251)$ nas mulheres.

Tabela I - Estado nutricional de adultos (20-59 anos) de um aglomerado urbano subnormal (Coelhos), segundo algumas variáveis biológicas e socioeconômicas. Recife-PE, 2014.

\begin{tabular}{|c|c|c|c|c|c|c|}
\hline \multirow{2}{*}{ Variáveis } & \multirow{2}{*}{$\begin{array}{c}\text { Baixo } \\
\text { peso } \\
\text { n (\%) }\end{array}$} & \multirow{2}{*}{$\begin{array}{c}\text { Adequado } \\
\mathbf{n}(\%)\end{array}$} & \multirow{2}{*}{$\begin{array}{c}\text { Sobrepeso } \\
\text { n (\%) }\end{array}$} & \multirow{2}{*}{$\begin{array}{c}\text { Obesidade } \\
\text { n (\%) }\end{array}$} & \multicolumn{2}{|c|}{ Total } \\
\hline & & & & & $\mathbf{n}$ & $\%$ \\
\hline \multicolumn{7}{|l|}{ Biológicas } \\
\hline \multicolumn{7}{|l|}{ Sexo } \\
\hline Masculino & $9(4,5)$ & $80(39,6)$ & $64(31,7)$ & $49(24,3)$ & 202 & 100,0 \\
\hline Feminino & $12(3,2)$ & $117(30,8)$ & $128(33,7)$ & $123(32,4)$ & 380 & 100,0 \\
\hline \multicolumn{7}{|l|}{ Faixa etária } \\
\hline $20-29$ & $13(9,2)$ & $62(43,7)$ & $41(29,9)$ & $26(18,3)$ & 142 & 100,0 \\
\hline $30-39$ & $1(0,7)$ & $47(31,8)$ & $56(37,8)$ & $44(29,7)$ & 148 & 100,0 \\
\hline $40-49$ & $5(3,0)$ & $45(27,4)$ & $60(36,6)$ & $54(32,9)$ & 164 & 100,0 \\
\hline $50-59$ & $2(1,6)$ & $43(33,6)$ & $35(27,3)$ & $48(27,3)$ & 128 & 100,0 \\
\hline \multicolumn{7}{|l|}{ Raça/cor } \\
\hline Branca & $3(2,8)$ & $41(37,6)$ & $29(26,6)$ & $36(33,0)$ & 109 & 100,0 \\
\hline Preta & $6(6,4)$ & $24(25,5)$ & $34(36,2)$ & $30(31,9)$ & 94 & 100,0 \\
\hline Parda & $12(3,3)$ & $126(34,5)$ & $122(33,4)$ & $105(28,8)$ & 365 & 100,0 \\
\hline \multicolumn{7}{|l|}{ Socioeconômicas } \\
\hline \multicolumn{7}{|l|}{ Condição de trabalho } \\
\hline Não trabalha ${ }^{a}$ & $3(3,8)$ & $22(27,5)$ & $23(28,8)$ & $32(40,0)$ & 80 & 100,0 \\
\hline Desempregado $^{\mathbf{b}}$ & $6(5,1)$ & $41(34,7)$ & $41(34,7)$ & $30(25,4)$ & 118 & 100,0 \\
\hline Benefícios sociais ${ }^{\mathrm{c}}$ & $1(1,9)$ & $23(43,4)$ & $16(30,2)$ & $13(24,5)$ & 53 & 100,0 \\
\hline Autônomo (urbano ou rural) & $2(1,8)$ & $31(27,4)$ & $37(32,7)$ & $43(38,1)$ & 113 & 100,0 \\
\hline Empregado & $5(3,7)$ & $43(32,1)$ & $51(38,1)$ & $35(26,1)$ & 134 & 100,0 \\
\hline Trabalho esporádicod & $4(4,8)$ & $37(44,0)$ & $24(28,6)$ & $19(22,6)$ & 84 & 100,0 \\
\hline \multicolumn{7}{|l|}{ Escolaridade } \\
\hline \multirow{2}{*}{$\begin{array}{l}\text { Analfabeto/Fundamental } 1 \text { incompleto } \\
\text { Fundamental } 1 \text { completo/ Fundamental } 2 \\
\text { incompleto }\end{array}$} & $5(4,8)$ & $35(33,7)$ & $32(30,8)$ & $32(30,8)$ & 104 & 100,0 \\
\hline & $3(2,1)$ & $47(32,9)$ & $48(33,6)$ & $45(31,5)$ & 143 & 100,0 \\
\hline \multirow{2}{*}{$\begin{array}{l}\text { Fundamental completo/ Médio incompleto } \\
\text { Médio completo/ superior incompleto/superior } \\
\text { completo }\end{array}$} & $6(5,0)$ & $44(36,4)$ & $29(24,0)$ & $42(34,7)$ & 121 & 100,0 \\
\hline & $7(3,3)$ & $71(33,8)$ & $80(38,1)$ & $52(24,8)$ & 210 & 100,0 \\
\hline
\end{tabular}

a: Nunca trabalhou e donas de casa b: Já trabalhou, mas estava desempregado há 30 dias ou mais c: aposentado, pensionista, benefícios provisórios $\mathbf{d}$ :biscateiro/ambulante, trabalho voluntário 
Com relação à distribuição por faixas etárias, ressalta-se que $9,2 \%(n=13)$ das observações no grupo de 20-29 anos se enquadravam na condição de baixo peso, enquanto no intervalo de 40-49 anos, 69,5\% ( $\mathrm{n}=114)$ se enquadravam na categoria de sobrepeso/obesidade, representando os valores mais elevados dessas duas condições. Em termos de etnias, $59,6 \%(n=65)$ da cor branca tinha sobrepeso/obesidade, condição que prevalecia para $68,1 \%(\mathrm{n}=64)$ da cor preta.

No que concerne às condições socioeconômicas, quase $70 \%(\mathrm{n}=55)$ dos que não trabalhavam tinham sobrepeso ou obesidade, correspondendo praticamente à situação dos autônomos $(70,8 \% ; n=80)$, categoria em que o problema resultou nas maiores frequências. Já entre os beneficiários de serviços em promoção social, 54,7\% $(n=29)$ tinham sobrepeso ou obesidade. Em termos descritivos, a escolaridade, distribuída em quatro categorias, não apresentou resultados discrepantes na classificação do estado nutricional, destacando-se, entretanto, que a maior proporção de excesso de peso foi identificada entre os adultos com o segundo menor nível de escolaridade $(65,1 \%$; $\mathrm{n}=93$ ).

Tendo em conta, a partir dos resultados descritivos, que o excesso de peso, configurado no binômio sobrepeso/ obesidade, representa o principal problema nutricional da população estudada, fez-se um exercício analítico de fatores de risco (Odds Ratio) através de análises bivariadas (Tabela II). Demonstra-se, assim, que a chance de ocorrência do excesso de peso aumentou significativamente a partir dos

Tabela II - Excesso de peso de adultos (20-59 anos) de um aglomerado urbano subnormal (Coelhos), segundo algumas variáveis biológicas e socioeconômicas. Recife-PE, 2014.

\begin{tabular}{|c|c|c|c|c|c|}
\hline \multirow{2}{*}{ Variáveis } & \multirow{2}{*}{$\begin{array}{c}\text { Amostra } \\
\mathbf{n}\end{array}$} & \multicolumn{2}{|c|}{ Excesso de peso } & \multirow{2}{*}{$\begin{array}{c}\text { OddsRatio } \\
\text { IC } 95 \% \\
\end{array}$} & \multirow[t]{2}{*}{$\mathbf{p}^{*}$} \\
\hline & & $\mathbf{n}$ & $\%$ & & \\
\hline \multicolumn{6}{|l|}{ Biológicas } \\
\hline Sexo & 582 & & & & \\
\hline Masculino & 202 & 113 & 55,9 & 1,0 & $0,016^{* *}$ \\
\hline Feminino & 380 & 251 & 66,1 & $1,5(1,0-2,2)$ & \\
\hline Faixa etária (anos) & 582 & & & & \\
\hline $20-29$ & 142 & 67 & 47,2 & 1,0 & $0,000 * *$ \\
\hline $30-39$ & 148 & 100 & 67,6 & $2,3(1,4-3,8)$ & \\
\hline $40-49$ & 164 & 114 & 69,5 & $2,6(1,6-4,1)$ & \\
\hline $50-59$ & 128 & 83 & 64,8 & $2,1(1,3-3,4)$ & \\
\hline Raça/cor & 568 & & & & \\
\hline Branca & 109 & 65 & 59,6 & 1,0 & 0,440 \\
\hline Preta & 94 & 64 & 68,1 & $1,4(0,8-2,6)$ & \\
\hline Parda & 365 & 227 & 62,2 & $1,1(0,7-1,7)$ & \\
\hline \multicolumn{6}{|l|}{ Socioeconômicas } \\
\hline Condição de trabalho & 582 & & & & \\
\hline Trabalho esporádico ${ }^{\mathrm{d}}$ & 84 & 43 & 51,2 & 1,0 & $0,050 * *$ \\
\hline Não trabalha ${ }^{a}$ & 80 & 55 & 68,8 & $1,7(1,0-3,0)$ & \\
\hline Desempregado $^{\mathrm{b}}$ & 118 & 71 & 60,1 & $2,3(1,3-4,2)$ & \\
\hline Benefícios Sociais ${ }^{\mathrm{c}}$ & 53 & 29 & 54,7 & $1,2(0,6-2,3)$ & \\
\hline Autônomo (urbano ou rural) & 113 & 80 & 70,8 & $1,4(0,8-2,5)$ & \\
\hline Empregado & 134 & 86 & 64,2 & $2,1(1,1-4,0)$ & \\
\hline Escolaridade & 578 & & & & \\
\hline $\begin{array}{l}\text { Médio completo/superior } \\
\text { incompleto/superior completo }\end{array}$ & 210 & 132 & 62,9 & 1,0 & 0,757 \\
\hline Fundamental completo/médio incompleto & 121 & 71 & 58,7 & $0,9(0,6-1,5)$ & \\
\hline Fundamental 1 completo/ fundamental 2 incompleto & 143 & 93 & 65,0 & $1,1(0,7-1,7)$ & \\
\hline Analfabeto/Fundamental 1 incompleto & 104 & 64 & 61,5 & $0,8(0,5-1,3)$ & \\
\hline
\end{tabular}

IC95\%: intervalo de confiança $=95 \% *$ Qui-quadrado de Pearson **p $(<0,05)$

a: Nunca trabalhou e donas de casa b: Já trabalhou, mas estava desempregado há 30 dias ou mais c: aposentado, pensionista, benefícios provisórios $\mathbf{d}$ :biscateiro/ambulante, trabalho voluntário 
30 anos de idade, elevando-se de $47,2 \%(n=67)$ para $67,6 \%$ $(n=100)$ e $69,5 \%(n=114)$, respectivamente, na faixa etária de 30-39 anos e de 40-49 anos ( $<<0,001)$. O sexo feminino comportou-se como outro fator biológico de risco (OR de 1,5). As condições de não trabalhar (OR 1,7; IC95:1,0-3,0), de estar desempregado (OR 2,3; IC95:1,3-4,2) e empregado (OR 2,1; IC95: 1,1-4,0) foram os fatores estatisticamente significantes com o risco de ocorrência do sobrepeso/ obesidade, tendo como referência o grupo "trabalho esporádico".

\section{DISCUSSÃO}

O estudo aqui relatado constitui uma das abordagens pioneiras, no sentido de analisar a situação nutricional de adultos num espaço urbano caracteristicamente pobre, como é o caso de populações radicadas em áreas faveladas, onde habitam, no Brasil, cerca de 12 milhões de pessoas ${ }^{(19)}$. Nesse sentido, é surpreendente verificar que o perfil epidemiológico evidenciado na amostra estudada não difere significativamente das estatísticas relatadas para populações urbanas do Brasil, seja no que se refere à condição de baixo peso ("proxi" da desnutrição energético-proteico ou DEP), seja no que se aplica ao problema oposto, ou seja, à condição de excesso de peso, compreendendo o sobrepeso e a obesidade. De fato, o inquérito mais recente avaliando o estado nutricional da população adulta urbana no Brasil, a Pesquisa de Orçamento Familiar 2008-2009 (POF) ${ }^{(24)}$, demonstra que $2,7 \%$ se enquadram na condição de baixo peso. Por outro lado, considerando o binômio sobrepeso/ obesidade, as prevalências nacionais descritas pela POF relatam a ocorrência de $62,6 \%$ para os homens e $64,9 \%$ para as mulheres. Em nível internacional, aceita-se que os Estados Unidos apresentam as estatísticas mais elevadas de excesso de peso em adultos com idade igual ou maior de 20 anos (70,9\% em homens e $61,9 \%$ em mulheres), seguindo-se a Austrália (68,6\% e 56,7\%), respectivamente, para homens e mulheres adultos. Já o México apresentava, seguindo a ordem de citação, prevalências de $66,8 \%$ e $71,4 \%{ }^{(7)}$.

Evidencia-se, nesse breve painel epidemiológico, que os valores encontrados na comunidade dos Coelhos praticamente se equivalem aos resultados obtidos em nível internacional e nacional, em inquéritos representativos da população. Essa observação é de grande importância, não apenas para o caso restrito da população favelada, mas por evidenciar que o parâmetro mais característico da transição nutricional (a pandemia do sobrepeso/ obesidade) não se restringe apenas ao contexto mais geral do Brasil e do mundo, estendendo-se também aos nichos ou ecossistemas de pobreza. É um processo que se difunde de forma homogênea mesmo para as populações circunscritas às condições socioeconômicas e ambientais mais adversas, como é o caso de famílias faveladas ${ }^{(12,25)}$.
E ainda que esses dados sejam de validade limitada, é interessante sua notificação preliminar como indicação de uma possível tendência mais geral: a homogeneização do cenário epidemiológico da população brasileira. Ou seja, a tendência de equalização do problema em todos os espaços geográficos (regional, estadual, macro, e microrregional) e em todos os estratos sociais.

Considerando, num recorte mais restrito e conceitualmente mais apropriado, a situação nutricional de populações faveladas, duas contribuições podem ser comparadas, como outro estudo ${ }^{(12)}$, num assentamento urbano subnormal de Maceió-AL, demonstrando que $41,2 \%$ dos adultos apresentaram sobrepeso ou obesidade. Trata-se, portanto, de uma prevalência bem mais baixa que a detectada em nosso estudo. Também em moradores de favelas, em São Paulo, observou-se que 36,5\% dos adultos apresentaram, cumulativamente, classificações de sobrepeso e obesidade ${ }^{(26)}$. Esse estudo é de 1995, sendo provável que a prevalência atual de excesso de peso seja bem mais elevada, considerando as tendências temporais de evolução do problema no Brasil.

Outro enfoque seria a avaliação de alguns fatores que se associam ao excesso de peso como condição de risco. Nessa perspectiva, não se dispõe de contribuições analíticas sistematizadas para compreender o problema no âmbito de assentamentos urbanos subnormais no Brasil, de forma que as referências aqui mencionadas foram retiradas de estudos com uma abrangência muito genérica.

Observou-se em nosso estudo que as proporções de sobrepeso e obesidade aumentaram com a idade, atingindo os maiores valores até a idade de 49 anos. Por diferentes razões biológicas e comportamentais, a ocorrência de excesso de peso tende a aumentar com a idade, com um possível ponto de inflexão situado entre 50-59 anos. Essa tendência genérica apresentou mudanças de ritmo e resultados finais, no Brasil, nas duas décadas mais recentes, em função da transição nutricional que modificou de forma marcante o perfil de morbimortalidade de nossa população ${ }^{(7)}$.

Na realidade, a relação do risco de sobrepeso/obesidade comoaumentodaidadeéuma observaçãode caráteruniversal, com raras exceções. Um estudo realizado na Colômbia descreve que a obesidade aumenta substancialmente com a idade ${ }^{(27)}$. Tais resultados também estão em concordância com os dados obtidos pela Vigilância de Fatores de Risco e Proteção para Doenças Crônicas por Inquérito Telefônico (VIGITEL) $^{(10)}$, os quais registraram que a frequência de sobrepeso tende a aumentar com a idade, até os 54 anos, em ambos os sexos. É um resultado consistente com outros estudos que apontam uma relação direta entre o aumento do peso e a idade do indivíduo ${ }^{(11,12,28,29)}$. A associação positiva entre a idade e o excesso de peso poderia ser explicada, em parte, pelo declínio da taxa metabólica que fisiologicamente 
acompanha o processo de envelhecimento e pela redução habitual da prática de atividades físicas ${ }^{(30)}$.

A maior prevalência de excesso de peso entre mulheres é um evento de abrangência mundial com raras exceções ${ }^{(7)}$. No caso do Brasil, o acompanhamento de uma série histórica iniciada em 1974 indica que o ritmo temporal de aumento do sobrepeso/obesidade tem sido cerca de três vezes maior entre os homens e de duas vezes entre as mulheres ${ }^{(24)}$. Em estudo realizado no Peru, observou-se que o excesso de peso foi um pouco maior nos homens que nas mulheres, mas especificamente a obesidade foi mais elevada em mulheres ${ }^{(31)}$ Uma pesquisa $^{(32)}$ com famílias pauperizadas, cadastradas no Programa Comunidade Solidária do Governo Brasileiro, identificou que 54,5\% das mulheres e $30,7 \%$ dos homens estavam com sobrepeso ou obesidade. Em população de baixa renda no Nordeste ${ }^{(12)}$ também encontraram uma frequência de excesso de peso maior entre as mulheres $(46,2 \%)$ do que entre os homens (32,6\%). Em Ribeirão Preto-SP ${ }^{(33)}$, identificaram maior prevalência de sobrepeso entre os homens, enquanto a obesidade prevalecia entre as mulheres.

A inexistência de associação entre o excesso de peso e a condição étnica (cor/raça) também foi encontrada em estudo de adultos em Salvador ${ }^{(34)}$ e Bahia ${ }^{(28)}$, em pesquisa com a população de 20 a 59 anos, no município de Santo Ângelo-RS. Isso expressa que os possíveis efeitos de relações preconceituosas, levando à discriminação no mercado de bens e serviços, já não se manifesta a nível de comunidade, produzindo implicações desfavoráveis ao estado de nutrição próteico-calórica.

Com relação à escolaridade, observou-se no presente estudo que a prevalência de obesidade aumentou à medida que o grau de instrução se elevou, até a categoria fundamental completo/médio incompleto, quando a tendência é interrompida. Esse resultado se assemelha ao outro $^{(35)} \mathrm{com}$ a população adulta beneficiária do Programa Bolsa Família no município de Curitiba-PR. Isso pode sugerir que, embora a população obtenha maior grau de instrução, a baixa condição socioeconômica favorecia a aquisição de alimentos mais baratos e mais calóricos, contribuindo para a maior frequência de obesidade. O VIGITEL ${ }^{(10)}$ obteve resultado diferente, revelando que a frequência de obesidade tende a diminuir com o aumento de escolaridade, demonstrando que $14,3 \%$ das pessoas com 12 ou mais anos de escolaridade tinham obesidade, contrapondo-se aos níveis de escolaridade mais baixos.

No presente estudo, não se observou associação estatisticamente significativa entre escolaridade e excesso de peso, corroborando com outro realizado no município de Santo Ângelo(RS) ${ }^{(28)}$. Em nível internacional, ressalta-se a importância da associação da escolaridade com o excesso de peso, como no estudo com adultos da Finlândia ${ }^{(36)}$ e na
Colômbia ${ }^{(27)}$. Já no Brasil, essa condição foi identificada no estudo em Ribeirão Preto-SP ${ }^{(33)}$ e no realizado com mulheres de baixa renda em Duque de Caxias- $\mathrm{RJ}^{(37)}$.

A variável "condição de trabalho" foi considerada uma variável associada ao excesso de peso, em razão de sua importância epidemiológica, como possível determinante de doenças e por apresentar um valor p próximo à "condição de significância" $(0,050)$. Em estudo de base populacional em Rio Branco-AC ${ }^{(38)}$, identificou-se a associação entre a condição de trabalho e o excesso de peso, concordando com o estudo aqui apresentado. Esses resultados, entretanto, diferem dos estudos realizados em adultos nas cidades de São Paulo ${ }^{(33)}$ e Maceió ${ }^{(12)}$. Eles constataram que o grupo de casos não inseridos no mercado de trabalho apresentou prevalência de excesso de peso de maior magnitude que aqueles que referiram ocupação no momento da entrevista.

Os autores reconhecem as limitações do estudo, que deve ser entendido como uma abordagem ainda preliminar de um projeto de longa duração a ser desenvolvido nesta comunidade: uma coorte de 10 anos de duração. Ressalta-se como limitação principal o inventário analítico do problema do sobrepeso/obesidade, restrito a apenas cinco grupos de variáveis explanatórias. Sem dúvida, a compreensão do excesso de peso demanda, como problema multifatorial, uma abordagem bem mais complexa, o que, por sinal, não constitui o objetivo dominante de nosso estudo. Ademais, prepondera uma limitação fundamental de caráter epistemológico, ou seja, como abordagem transversal, efeitos e possíveis antecedentes causais são tomados simultaneamente, desconsiderando a relação antes/depois que, por lógica formal, deve condicionar a relação.

\section{CONCLUSÃO}

A elevada prevalência do excesso de peso na população adulta residente no aglomerado urbano subnormal dos Coelhos se mostrou associada ao sexo, faixa etária e condição de trabalho, constituindo-se, assim, como possíveis fatores de risco do problema.

\section{AGRADECIMENTOS}

Ao professor da Universidade Federal de Pernambuco, Pedro Israel Cabral de Lira, lotado no Departamento de Nutrição da Universidade Federal de Pernambuco, pelo apoio na confecção do banco de dados e análise dos dados.

\section{REFERÊNCIAS}

1. Ministério da Saúde (BR), Secretaria de Atenção à Saúde, Departamento de Atenção Básica. Diretrizes para o cuidado das pessoas com doenças crônicas 
nas redes de atenção à saúde e nas linhas de cuidado prioritárias. Brasília: Ministério da Saúde; 2013.

2. Ministério da Saúde (BR), Secretária de Vigilância em Saúde, Departamento de Análise de Situação de Saúde. Saúde Brasil 2010: uma análise da situação de saúde e de evidências selecionadas de impacto de ações de vigilância em saúde. Brasília: Ministério da Saúde; 2011.

3. Batista Filho M, Rissin A. A transição nutricional no Brasil: tendências regionais e temporais. Cad Saúde Pública. 2003;19 (Supl 1):S181-91.

4. Freese E, Fontbonne A. Transição epidemiológica comparada: modernidade, precariedade e vulnerabilidade. In: Freese E, organizador. Epidemiologia, políticas e determinantes das doenças crônicas não transmissíveis no Brasil. Recife: Ed. Universitária da UFPE; 2006. p. 17-46.

5. World Health Organization - WHO. Global status report on noncommunicable diseases, 2010 [acesso em 2014 Set 26]. Disponível em: http://www.who.int/nmh/ publications/ncd_report_full_en.pdf

6. Goulart FAA. Doenças crônicas não transmissíveis: estratégias de controle e desafios para os sistemas de saúde. Brasília: Organização Pan-Americana da Saúde; 2011.

7. Ng M, Fleming T, Robinson M, Thomson B, Graetz $\mathrm{N}$, Margono $\mathrm{C}$, et al. Global, regional, and national prevalence of overweight and obesity in children and adults during 1980-2013: a systematic analysis for the Global Burden of Disease Study 2013. Lancet. 2014;384(9945):766-81

8. Schmidt MI, Duncan BB, Silva GA, Menezes MA, Monteiro MA, Barreto SM, et al. Doenças crônicas não transmissíveis no Brasil: carga e desafios atuais. Lancet Saúde no Brasil 4. [Internet]. 2014 [acesso em 2014 Set 26]. Disponível em: http://download.thelancet.com/ flatcontentassets/pdfs/brazil/brazilpor4.pdf

9. Mendes EV. O cuidado das condições crônicas na atenção primária à saúde: o imperativo da consolidação da estratégia da saúde da família. Brasília: Organização Pan-Americana da Saúde, 2012.

10. Ministério da Saúde (BR), Secretaria de Vigilância em Saúde. VIGITEL Brasil 2013: Vigilância de Fatores de Risco e Proteção para Doenças Crônicas por Inquérito Telefônico. Brasília: Ministério da Saúde; 2014.

11. Sá NN, Moura EC. Excesso de peso: determinantes sociodemográficos e comportamentais em adultos, Brasil, 2008. Cad Saúde Pública. 2011;27(7):1380-92.
12. Barbosa JM, Cabral PC, Lira PI, Florêncio TM. Fatores socioeconômicos associados ao excesso de peso em população de baixa renda do nordeste brasileiro. Arch Latinoam Nutr. 2009;59(1):22-9.

13. Ogden CL, Carroll MD, Kit BK, Flegal KM. Prevalence of Obesity in the United States, 2009-2010. NCHS Data Brief. 2012;82:1-8.

14. World Health Organization - WHO. Obesity and overweight [acesso em 2014 Set 22]. Disponível em: http://www.who.int/mediacentre/factsheets/fs311/en/

15. Gigante DP, de Moura EC, Sardinha LM. Prevalence of overweight and obesity and associated factors, Brazil. Rev Saúde Pública. 2009;43(Supl 2):83-9.

16. Kain J, Vio F, Albala C. Obesity trends and determinant factors in Latin America. Cad Saúde Pública. 2003;19(Supl 1):77-86.

17. Ferreira VA, Silva AE, Rodrigues CA, Nunes NL, Vigato TC, Magalhães R. Desigualdade, pobreza e obesidade. Ciênc Saúde Coletiva. 2010;15(Supl 1):1423-32

18. Malta DC, Silva JB. O Plano de Ações Estratégicas para o Enfrentamento das Doenças Crônicas Não Transmissíveis no Brasil e a definição das metas globais para o enfrentamento dessas doenças até 2025: uma revisão. Epidemiol Serv Saúde. 2013;22(1):15164.

19. Instituto Brasileiro de Geografia e Estatística - IBGE. Aglomerados subnormais Primeiros resultados. Rio de Janeiro: IBGE; 2010.

20. Silva CV, Zaffari D. Prevalência de excesso de peso e associação com outras variáveis em indivíduos adultos atendidos em unidade básica de saúde. Sci Med. 2009;19(1):17-26.

21. Ferreira HS, Florêncio TM, Fragoso MA, Melo FP, Silva TG. Hipertensão, obesidade abdominal e baixa estatura: aspectos da transição nutricional em uma população favelada. Rev Nutr. 2005;18(2):209-18.

22. Universidade Federal de Pernambuco, Centro de Ciências da Saúde, Departamento de Nutrição. III Pesquisa estadual de saúde e nutrição: saúde, nutrição, alimentação, condições socioeconômicas e atenção à saúde no estado de Pernambuco, 2006. Recife: UFPE; 2012.

23. World Health Organization - WHO. Physical status: the use and interpretation of antropometry. Geneva: WHO; 1995.

24. Instituto Brasileiro de Geografia e Estatística IBGE. Pesquisa de orçamentos familiares 2008- 
2009: antropometria e estado nutricional de crianças, adolescentes e adultos no Brasil. Rio de Janeiro: IBGE; 2010

25. Ministério da Saúde (BR). Como está sua saúde: resultados da Pesquisa sobre a situação de saúde dos moradores da região de Manguinhos. Rio de Janeiro: Fundação Oswaldo Cruz; 2013. (Boletim 1).

26. Sawaya AL, Dallal G, Solymos G, Sousa MH, Ventura ML, Roberts SB, et al. Obesity and malnutrition in a shantytown population in the city of São Paulo, Brazil. Obes Res. 1995;3(Suppl 2):107-15.

27. Castaño LSA, Rueda JDG, Aguirre CC. Factoressociales y económicos asociados a laobesidad: losefectos de lainequidad y de la pobreza. Rev Gerenc Politicas Salud. 2012;11(23):98-110.

28. Sarturi JB, Neves J, Peres KG. Obesidade em adultos: estudo de base populacional num município de pequeno porte no sul do Brasil em 2005. Ciênc Saúde Coletiva. 2010;15(1):105-13.

29. Holanda LGM, Martins MCC, Souza Filho MD, Carvalho CMRG, Assis RC, Leal LMM, et al. Excesso de peso e adiposidade central em adultos de Teresina-PI. AMB Rev Assoc Med Bras. 2011;57(1):50-5.

30. Francischi RPP, Pereira LO, Freitas MS, Klopfer M, Santos RC, Vieira O, et al. Obesidade: atualização sobre sua etiologia, morbidade e tratamento. Rev Nutr. 2000;13(1):17-28.

31. Dongo DA, Abanto JS, Guizado GG, Mamani1 CT. Sobrepeso y obesidad: prevalencia y determinantes socialesdelexceso de peso enlapoblación peruana (2009-2010). Rev Peru Med Exp Salud Publica. 2012;29(3):303-13.

32. Marinho SP, Martins IS, Perestrelo JPP, Oliveira DC. Obesidade em segmentos pauperizados da sociedade. Rev Nutr. 2003;16(2):195-201.
33. Moraes AS, Humberto JSM, Freitas ICM. Estado nutricional e fatores sociodemográficos em adultos residentes em Ribeirão Preto, SP, 2006: Projeto OBEDIARP. Rev Bras Epidemiol. 2011;14(4):662-76.

34. Oliveira LPM, Assis AMO, Silva MCMS, Santana MLP, Santos NS, Pinheiro SM, et al. Fatores associados a excesso de peso e concentração de gordura abdominal em adultos na cidade de Salvador, Bahia, Brasil.Cad Saúde Pública. 2009;25(3):570-82.

35. Lima FEL, Rabito EI, Dias MRMG. Estado nutricional de população adulta beneficiária do Programa Bolsa Família no município de Curitiba, PR. Rev Bras Epidemiol. 2011;14(2):198-206.

36. Klumbiene J, Petkeviciene J, Helasoja V, Prättälä R, Kasmel A. Sociodemographic and health behaviourfactors associated with obesity in adult populations in Estonia, Finland and Lithuania. Eur $\mathrm{J}$ Public Health. 2004;14(4):390-4.

37. Lins APM, Sichieri R, Coutinho WF, Ramos EG, Peixoto MVM, Fonseca VM. Alimentação saudável, escolaridade e excesso de peso entre mulheres de baixa renda. Ciênc Saúde Coletiva. 2013;18(2):357-66.

38. Lino MZR, Muniz PT, Kamile SS. Prevalência e fatores associados ao excesso de peso em adultos: inquérito populacional em Rio Branco, Acre, Brasil, 2007-2008. Cad Saúde Pública. 2011;27(4):797-810.

\section{Endereço para correspondência:}

Silvia Pereira da Silva de Carvalho Melo

Centro de Pesquisas Aggeu Magalhães (CPqAM)

Fundação Oswaldo Cruz - Pernambuco

Av. Professor Moraes Rego, s/n

Campus da UFPE - Cidade Universitária

CEP: 50.740-465 - Recife - PE - Brasil

E-mail: silviaps2008@gmail.com 